\title{
Review of:
}

\section{c-Myc suppresses $\mathrm{P} 21^{\mathrm{WAF} 1 / \mathrm{CIP} 1}$ expression during oestrogen signalling and antioestrogen resistance in human breast cancer cells}

\author{
C. M. McNeil, E. A. Musgrove \\ Cancer Research Program, Garvan Institute of Medical Research, NSW, Australia.
}

\section{Citation of original article:}

S. Mukherjee, S. E. Conrad. Journal of Biological Chemistry 2005; 280: 17616-17625.

\begin{abstract}
of the original article:
Oestrogen rapidly induces expression of the proto-oncogene c-Myc. c-Myc is required for oestrogen-stimulated proliferation of breast cancer cells, and deregulated c-Myc expression has been implicated in antioestrogen resistance. In this report, we investigate the mechanism(s) by which c-Myc mediates oestrogen-stimulated proliferation and contributes to cell cycle progression in the presence of antioestrogen. The MCF-7 cell line is a model of oestrogen-dependent, antioestrogen-sensitive human breast cancer. Using stable MCF-7 derivatives with inducible c-Myc expression, we demonstrated that in antioestrogen-treated cells, the elevated mRNA and protein levels of $\mathrm{p} 21^{\mathrm{WAF} 1 / \mathrm{CIP} 1}$, a cell cycle inhibitor, decreased upon either $\mathrm{c}-\mathrm{Myc}$ induction or oestrogen treatment. Expression of p21 blocked c-Myc-mediated cell cycle progression in the presence of antioestrogen, suggesting that the decrease in $\mathrm{P} 21^{\mathrm{WAF} 1 / \mathrm{CIP} 1}$ is necessary for this process. Using RNA interference to suppress c-Myc expression, we further established that c-Myc is required for oestrogen-mediated decreases in $\mathrm{p} 21^{\mathrm{WAF} 1 / \mathrm{CIP} 1}$. Finally, we observed that neither $\mathrm{c}-\mathrm{Myc}$ nor $\mathrm{p} 21^{\mathrm{WAF} 1 / \mathrm{CIP} 1}$ is regulated by oestrogen or antioestrogen in an antioestrogen-resistant MCF-7 derivative. The p21 levels in the antioestrogen-resistant cells increased when c-Myc expression was suppressed, suggesting that loss of p21 regulation was a consequence of constitutive c-Myc expression. Together, these studies implicate $\mathrm{p} 21^{\mathrm{WAF} 1 / \mathrm{CIP} 1}$ as an important target of c-Myc in breast cancer cells and provide a link between oestrogen, c-Myc, and the cell cycle machinery. They further suggest that aberrant c-Myc expression, which is frequently observed in human breast cancers, can contribute to antioestrogen resistance by altering $\mathrm{P} 21^{\mathrm{WAF} 1 / \mathrm{CIP} 1}$ regulation.
\end{abstract}

\section{Review}

While the hormonal treatment of oestrogen receptor (ER)-positive tumours has been a major advance in

Correspondence to: C. M. McNeil MBBS, Garvan Institute of Medical Research, 384 Victoria St, Darlinghurst, NSW, 2010, Australia. E-mail: c.mcneil@garvan.org.au; Tel: +612 9295 8322; Fax: +612 92958321

Received: 25/07/05

Accepted: 11/08/05

First published online 29/03/06

BCO/492/2005/JC the treatment of breast cancer, a significant percentage of hormone receptor positive breast cancers are inherently antioestrogen resistant, or become antioestrogen resistant during treatment [1,2]. An understanding of how antioestrogens act and how resistance may develop is an important goal of breast cancer research. To date aberrations in a number of signalling pathways have provided potential mechanisms for antioestrogen resistance. These include cross-talk between the ER and cell-surface tyrosine 
kinase receptors, as well as alterations in the MAP kinase and PI3-kinase pathways [1,2]. An important focus of study has understandably been at the point of convergence of these mitogenic signalling pathways, that is control of cell proliferation, where the transcription factor c-Myc is an important player [3].

Studies in clinical cohorts have failed to demonstrate a relationship between c-Myc amplification or c-Myc expression and overall outcome, including response to therapy, in breast cancer [4,5]. This may relate to the wide variability of amplification and expression levels identified across different cohorts as a consequence of variation in experimental techniques, contamination by non-tumour cells, and heterogeneous study populations, as well as an absence of data pertaining to patient follow-up, tumour characteristics and treatment $[4,5]$. Nonetheless there are significant in vitro data implicating C-Myc in breast cancer cell proliferation, and evidence suggesting that it may also be involved in the development of antioestrogen resistance. Regulation of c-Myc is an early response to either oestrogen or antioestrogen treatment of breast cancer cells [6]. Overexpression of c-Myc reverses or attenuates antioestrogen inhibition of breast cancer cell proliferation $[7,8]$, and antisense oligonucleotide-mediated suppression of c-Myc expression leads to an inhibition of proliferation that is accompanied by molecular changes that mimic the effects of antioestrogens [9].

In their recent article, Mukherjee and Conrad provide further mechanistic insight into the potential role of c-Myc in resistance to antioestrogens in breast cancer cells in vitro [10]. Consistent with a previous study using MCF-7 cells [7], inducible expression of c-Myc led to re-initiation of cell cycle progression in antioestrogen-arrested cells, although this was less effective than oestrogen treatment [10]. Thus, there appears to be a link between c-Myc overexpression and resistance to antioestrogens. Overall, the kinetics of cell cycle progression in response to oestrogen treatment or c-Myc induction were similar. However, some differences were apparent, particularly at later time points in the analysis, which may relate to differences in the magnitude and/or time course of c-Myc induction. However, it is also possible that they resulted from effects of oestrogen treatment that were independent of c-Myc, for example induction of cyclin D1 and activation of cyclin D1-CDK4 [7].

Consistent with previous data in which c-Myc decreased the transcription of the CDK inhibitor p21 WAF1/CIP1 $(p 21)$ in other cell types [11-13], Mukherjee and Conrad demonstrated that decreased p21 expression was a pronounced response to c-Myc induction in MCF-7 cells [10]. Other studies have shown that oestrogen also decreased p21 transcription during re-initiation of cell cycle progression in antioestrogen-arrested cells and this was necessary for oestrogen-mediated CDK activation [14-17]. In addition, decreased p21 expression conferred resistance to the anti-proliferative effects of antioestrogens $[18,19]$ and increased p21 expression inhibited the ability of oestrogen to overcome antioestrogen-mediated arrest [20]. Similarly, when Mukherjee and Conrad overexpressed p21 using a recombinant adenovirus, c-Myc could not promote proliferation in antioestrogen-arrested cells [10]. Furthermore, RNA interference experiments showed that when c-Myc expression was decreased, p21 expression remained high in the presence of oestrogen [10]. Collectively, these observations suggest that the ability of oestrogen to decrease p21 transcription might be mediated by c-Myc.

Mukherjee and Conrad used luciferase reporter assays to show that a fragment of the p21 promoter beginning at $-194 \mathrm{bp}$, encompassing a region previously shown to bind c-Myc [13], was repressed by c-Myc in MCF-7 cells [10], with the implication that oestrogen/antioestrogen-mediated regulation of c-Myc would lead to regulation of p21 via the same promoter element. Others have localised the sequence required for increased p21 promoter activity in response to antioestrogen treatment to a similar region, between -143 and +8 [21]. Mutation of individual Sp1 sites within this region substantially attenuated the induction, and further assays including chromatin immunoprecipitation suggested a model in which an ER-Sp1 complex binds to this region in cycling cells, recruiting histone deacetylases that repress p21 transcription [21]. Treatment with the antioestrogen ICI 182,780 led to disruption of this association and enhanced recruitment of Sp1 to the p21 promoter [21]. Gartel et al [12] demonstrated that c-Myc bound Sp1 but did not directly interact with the p21 promoter, suggesting that c-Myc may repress p21 by titrating Sp1 away from the p21 promoter. In hormone-responsive cells, ER and c-Myc may collectively modulate Sp1 regulation of p21 transcription. Thus although the experiments of Mukherjee and Conrad suggest that oestrogen/antioestrogen effects on p21 transcription are primarily mediated by $\mathrm{c}-\mathrm{Myc}$, the precise mechanism by which this occurs requires further study.

An increase in p21 levels when c-Myc is decreased has not been observed in another study using MCF-7 cells [9], perhaps due to differences in experimental design, whereby p21 expression was evaluated after 8-16 h of antisense c-Myc-oligonucleotide treatment as compared to $48 \mathrm{~h}$ of c-Myc RNA interference. However, despite the apparent differences between these studies, the overall conclusion is that regulation of p21 expression is central to the ability of c-Myc to 
modulate cell cycle progression in these cells, since overexpression of p21 using a recombinant adenovirus abrogated the ability of c-Myc to induce proliferation in antioestrogen-arrested cells [10], and decreased p21 expression allowed continued proliferation despite decreased c-Myc expression [9].

In a further set of experiments Mukherjee and Conrad investigated the potential role of c-Myc and p21 in resistance to antioestrogens in LCC9 breast cancer cells. These cells are an ER-positive, but oestrogen-independent and antioestrogen-resistant derivative of MCF-7 cells. They displayed no alteration in c-Myc or p21 expression after oestrogen or antioestrogen treatment [10]. When c-Myc was suppressed in LCC9 cells using RNA interference, p21 expression increased after antioestrogen treatment. It has been shown elsewhere that proliferation of antioestrogen-sensitive cells continues in the face of antioestrogen treatment if the increase in p21 is prevented using antisense oligonucleotides, or if c-Myc expression is maintained $[8,18,19]$. Collectively, these data suggest that the antioestrogen resistance observed in LCC9 cells may be due to altered p21 regulation by c-Myc.

The potential clinical importance of this article lies in its description of a mechanism through which c-Myc may mediate antioestrogen resistance. The questions that now arise include whether p21 modulation accounts for the majority of the antioestrogen resistance associated with c-Myc overexpression, and whether other upstream mediators are also involved. Although a previous study suggested that c-Myc induction resulted in cell cycle progression equivalent to the oestrogen response [7], Mukherjee and Conrad concluded that c-Myc induction alone was less effective than oestrogen treatment. Other investigators have demonstrated that c-Myc confers only partial antioestrogen resistance [8]. Thus the quantitative relationship between the level of c-Myc expression and oestrogen/antioestrogen sensitivity, and whether there is a level of c-Myc that can confer complete antioestrogen resistance, is unclear. An additional question relates to the degree to which c-Myc expression may influence antioestrogen resistance in the clinic. Further studies are required to dissect out other downstream targets of c-Myc with potential roles in steroid sensitivity, and upstream influences on p21 activity in order to direct research towards future therapeutic strategies for women with antioestrogenresistant breast cancers.

Thus the important mechanistic study of Mukherjee and Conrad provides a framework for further testing these hypotheses in the laboratory and in tumour material from women with known therapeutic response to endocrine therapies.

\section{Acknowledgments}

Dr. McNeil is supported by the National Health and Medical Research Council, and the Cancer Institute of New South Wales. Associate Professor Musgrove is supported by the National Health and Medical Research Council, The Cancer Council NSW, the Association for International Cancer Research and is a Cancer Institute of New South Wales Fellow.

\section{References}

1. Osborne CK, Shou J, Massarweh S, Schiff R. Crosstalk between estrogen receptor and growth factor receptor pathways as a cause for endocrine therapy resistance in breast cancer. Clin Cancer Res 2005; 11: 865s-870s.

2. Nicholson RI, Staka C, Boyns F, Hutcheson IR, Gee JM. Growth factor-driven mechanisms associated with resistance to estrogen deprivation in breast cancer: new opportunities for therapy. Endocr Relat Cancer 2004; 11: 623-641.

3. Adhikary S, Eilers M. Transcriptional regulation and transformation by Myc proteins. Nat Rev Mol Cell Biol 2005; 6: 635-645.

4. Deming SL, Nass SJ, Dickson RB, Trock BJ. C-myc amplification in breast cancer: a meta-analysis of its occurrence and prognostic relevance. Br J Cancer 2000; 83: 1688-1695.

5. Blancato J, Singh B, Liu A, et al. Correlation of amplification and overexpression of the c-myc oncogene in highgrade breast cancer: $\mathrm{FISH}$, in situ hybridisation and immunohistochemical analyses. Br J Cancer 2004; 90: 1612-1619.

6. Foster JS, Henley DC, Ahamed S, Wimalasena J. Estrogens and cell-cycle regulation in breast cancer. Trend Endocrinol Metab 2001; 12: 320-327.

7. Prall OW, Rogan EM, Musgrove EA, Watts CK, Sutherland RL. C-Myc or cyclin D1 mimics estrogen effects on cyclin E-Cdk2 activation and cell cycle reentry. Mol Cell Biol 1998; 18: 4499-4508.

8. Venditti M, Iwasiow B, Orr FW, Shiu RP. C-myc gene expression alone is sufficient to confer resistance to antiestrogen in human breast cancer cells. Int $J$ Cancer 2002; 99: 35-42.

9. Carroll JS, Swarbrick A, Musgrove EA, Sutherland RL. Mechanisms of growth arrest by c-myc antisense oligonucleotides in MCF-7 breast cancer cells: implications for the antiproliferative effects of antiestrogens. Cancer Res 2002; 62: 3126-3131.

10. Mukherjee S, Conrad SE. C-Myc suppresses p21WAF1/CIP1 expression during estrogen signaling and antiestrogen resistance in human breast cancer cells. J Biol Chem 2005; 208:17617-17625.

11. Coller HA, Grandori C, Tamayo P, et al. Expression analysis with oligonucleotide microarrays reveals that MYC regulates genes involved in growth, cell cycle, signaling, and adhesion. Proc Natl Acad Sci USA 2000; 97: 3260-3265.

12. Gartel $A L$, Ye X, Goufman E, et al. Myc represses the p21(WAF1/CIP1) promoter and interacts with Sp1/Sp3. Proc Natl Acad Sci USA 2001; 98: 4510-4515.

13. Seoane J, Le HV, Massague J. Myc suppression of the p21(Cip1) Cdk inhibitor influences the outcome of the 
p53 response to DNA damage. Nature 2002; 419: 729-734

14. Planas-Silva MD, Weinberg RA. Estrogen-dependent cyclin E-cdk2 activation through p21 redistribution. Mol Cell Biol 1997; 17: 4059-4069.

15. Prall OW, Carroll JS, Sutherland RL. A low abundance pool of nascent p21WAF1/Cip1 is targeted by estrogen to activate cyclin $E^{\star}$ Cdk2. J Biol Chem 2001; 276: 45433-45442.

16. Prall OW, Sarcevic B, Musgrove EA, Watts CK, Sutherland RL. Estrogen-induced activation of Cdk4 and Cdk2 during G1-S phase progression is accompanied by increased cyclin D1 expression and decreased cyclindependent kinase inhibitor association with cyclin E-Cdk2. J Biol Chem 1997; 272: 10882-10894.

17. Skildum AJ, Mukherjee S, Conrad SE. The cyclindependent kinase inhibitor p21WAF1/Cip1 is an antiestrogen-regulated inhibitor of Cdk4 in human breast cancer cells. J Biol Chem 2002; 277: 5145-5152.

18. Cariou S, Donovan JC, Flanagan WM, Milic A, Bhattacharya N, Slingerland JM. Down-regulation of
p21WAF1/CIP1 or p27Kip1 abrogates antiestrogenmediated cell cycle arrest in human breast cancer cells. Proc Natl Acad Sci USA 2000; 97: 9042-9046.

19. Carroll JS, Prall OW, Musgrove EA, Sutherland RL. A pure estrogen antagonist inhibits cyclin E-Cdk2 activity in MCF-7 breast cancer cells and induces accumulation of p130-E2F4 complexes characteristic of quiescence. J Biol Chem 2000; 275: 38221-38229.

20. Lai A, Sarcevic B, Prall OW, Sutherland RL. Insulin/insulin-like growth factor-I and estrogen cooperate to stimulate cyclin E-Cdk2 activation and cell cycle progression in MCF-7 breast cancer cells through differential regulation of cyclin $\mathrm{E}$ and $\mathrm{p} 21(\mathrm{WAF} 1 / \mathrm{Cip} 1)$. $J$ Biol Chem 2001; 276: 25823-25833.

21. Varshochi R, Halim F, Sunters A, Alao JP, Madureira PA, Hart SM, et al. ICl182,780 induces p21Waf1 gene transcription through releasing histone deacetylase 1 and estrogen receptor alpha from $\mathrm{Sp} 1$ sites to induce cell cycle arrest in MCF-7 breast cancer cell line. J Biol Chem 2005; 280: 3185-3196. 